\title{
DEGREE SEQUENCES OF INFINITE GRAPHS
}

\author{
ANDREAS BLASS AND FRANK HARARY
}

\section{ABSTRACT}

The degree sequences of finite graphs, finite connected graphs, finite trees and finite forests have all been characterized. Our present purpose is to provide such characterizations in the infinite case.

\section{Introduction and assumptions}

All graphs in this paper are without loops and multiple edges, as in [3], but they need not be finite. The degree of a vertex in a graph is the cardinality of its neighbourhood (the set of all vertices adjacent to it). A graph is locally finite if every vertex has finite degree. For a countable locally finite graph, the degrees of the vertices can be listed as a sequence of integers, called the degree sequence of the graph. The degree sequences of finite graphs of finite connected graphs, of finite trees, and of finite forests have been characterized by several authors, as documented in Hakimi and Schmeichel [1]. Our present purpose is to give such characterizations in the infinite case. We treat countable locally finite graphs in Section 1 and all other infinite graphs in Section 2.

Strictly speaking, the concept of degree sequence applies only to the countable locally finite case, though it could easily be generalized by allowing (possibly) transfinite sequences of (possibly) infinite cardinals. However, since degree sequences involve an ordering of the vertices (or at least of their degrees) that is entirely irrelevant to the problem under consideration, it is preferable to dispense with them, using instead the following construct. The multiplicity function $m$ of a graph assigns to each cardinal number $d$ the number $m(d)$ of vertices having degree $d$. (The domain of $m$ consists of all cardinal numbers, but $m(d)=0$ for all sufficiently large $d$, so we need not be concerned about any set-theoretic difficulties.) Thus, for example, the number of vertices of the graph is $\sum_{d} m(d)$, and the graph is locally finite if and only if $m(d)=0$ for all infinite $d$. We shall characterize those functions $m$ from cardinals to cardinals that occur as the multiplicity functions of infinite graphs, forests, trees, and connected graphs.

Three assumptions (A1-A3 below) about $m$ will be in force throughout the paper.

A1. $m(d)=0$ for all sufficiently large cardinal numbers $d$.

Clearly, the multiplicity function of any graph satisfies this requirement. A1 implies that the cardinal sum $\sum_{d} m(d)$ is well-defined; it is the number of vertices in any graph with multiplicity function $m$, and we denote it by $p$.

A2. $p$ is infinite.

This simply means that we are dealing with infinite graphs.

A3. $m(0)=0$.

Received 5 November, 1979.

[J. LONDON MATH. SOC. (2), 23 (1981), 10-16] 
This assumption entails no loss of generality, for if $m(0)>0$ then $m$ cannot be the multiplicity function of a connected graph, while the question whether $m$ is the multiplicity function of a graph or a forest reduces immediately to the same question for the function $m^{\prime}$ that agrees with $m$ except that $m^{\prime}(0)=0$.

\section{Countable locally finite graphs}

Throughout this section, we assume, in addition to our blanket assumptions A1A3, the following two conditions.

A4. $m(d)=0$ for all infinite $d$.

A5. $m(d) \leqslant \aleph_{0}$ for all $d$.

Thus, any graph with multiplicity function $m$ is countable and locally finite.

THEOREM 1. Every function satisfying A1-A5 is the multiplicity function of a forest.

THEOREM 2. For a function $m$ satisfying A1-A5, the following are equivalent:

(a) $m$ is the multiplicity function of a connected graph;

(b) $m$ is the multiplicity function of a tree;

(c) $1+\sum_{d \geqslant 3}(d-2) \cdot m(d) \geqslant m(1)$.

Before proving these two theorems, we introduce a way of viewing graphs that will be useful in describing the construction of graphs with prescribed multiplicity functions. Think of vertices as geometrical points and edges as (Jordan) arcs joining pairs of points, and think of each arc as being bisected, half of the arc being associated with each of the incident vertices. (This point of view is applied to digraphs in $[4,5]$.) Thus, we view the graph as being composed of "pieces", where each piece consists of a vertex of some degree $d$ together with its $d$ incident semiedges, and where the pieces are assembled into a graph by splicing together the open ends of certain pairs of semi-edges. We call such pieces, containing one vertex each, atoms, and we use the term semi-graph for the result of splicing some (or none or all) of the semi-edges of some collection of atoms in such a way as not to introduce loops or multiple edges. Thus, a semi-graph looks like a graph plus unspliced semiedges attached to some (or all or none) of the vertices.

This can be expressed formally by defining a semi-graph to be a triple $(G, H, \alpha)$, where $G$ is a graph, $H$ is a set called the set of (unspliced) semi-edges, and $\alpha$ is a function associating to every semi-edge in $H$ a vertex of $G$ said to be incident with that semi-edge. The degree of a vertex $v$ in this semi-graph is defined to be the sum of its degree in $G$ (called its spliced degree) and the number $\left|\alpha^{-1}\{v\}\right|$ of incident semiedges in $H$ (called its unspliced degree). To splice a pair of semi-edges in $H$ means to replace $(G, H, \alpha)$ with $\left(G^{\prime}, H^{\prime}, \alpha^{\prime}\right)$ where $H^{\prime}$ is $H$ minus the two semi-edges in question, $\alpha^{\prime}$ is $\alpha$ restricted to $H^{\prime}$, and $G^{\prime}$ is $G$ plus a new edge joining the vertices of those two semi-edges. Such splicing is possible only if these vertices are distinct and not adjacent in $G$. Similarly, one can define simultaneous splicing of several pairs of semi-edges. 
Given a proposed multiplicity function $m$, we have an associated semi-graph consisting of $m(d)$ vertices of unspliced degree $d$ and spliced degree 0 for every $d$ (a disjoint union of $p$ atoms). To construct a graph with multiplicity function $m$ is to splice all the semi-edges of this semi-graph. Such splicing will be carried out in stages to construct the required graphs in the proofs of Theorems 1 and 2, to which we now turn.

Proof of Theorem 1. Let $m$ be given, and let $P_{0}$ be the associated semi-graph as in the preceding paragraph. By assumptions A2-A5, $P$ has denumerably many vertices $v_{0}, v_{1}, \ldots$, and denumerably many semi-edges $s_{0}, s_{1}, \ldots$. We splice the semiedges together in denumerably many steps, splicing one pair of semi-edges at each step, as follows. Let $s_{n}$ be the first semi-edge (that is, with smallest subscript) not spliced at a previous stage; let $v_{k}$ be the first vertex that is distinct from $\alpha\left(s_{n}\right)$ and is not incident to any semi-edge already spliced. Then splice $s_{n}$ and an arbitrary semiedge incident to $v_{k}$. By the choice of $v_{k}$ and by $\mathrm{A} 3$ the splicing is possible, while the choice of $s_{n}$ ensures that every semi-edge is eventually spliced, so we will obtain a graph after countably many steps. Furthermore, the new edge formed at any stage of the construction cannot complete a cycle, for one of its endpoints was not previously joined to anything. Thus, no cycle is ever formed, and the construction yields a forest.

\section{Proof of Theorem 2. Obviously (b) implies (a).}

(c) $\Rightarrow$ (b). Assume that $m$ satisfies (c), and let $P_{0}$ be the associated semigraph, as in the proof of Theorem 1.

We shall first splice all but one of the (semi-edges of) vertices of degree 1 to (semiedges incident to) vertices of degree $\geqslant 3$ in such a way as to leave the unspliced degrees of the latter $\geqslant 2$. To do this, we set aside for each vertex of degree $\geqslant 3$, two of its incident semi-edges to be kept unspliced, and declare the rest available for splicing to vertices of degree 1 . There are $\sum_{d \geqslant 3}(d-2) \cdot m(d)$ available semi-edges, which, by (c), are enough to accommodate all but one of the $m(1)$ vertices of degree 1 .

Let the components of the resulting semi-graph be listed as $C_{0}, C_{1}, \ldots$, and let the unspliced semi-edges be $s_{0}, s_{1}, \ldots$ (Both sets are countable by A4 and A5.) If there is a component with only one unspliced semi-edge, then, by the preceding paragraph, there is only one such; let it be $C_{0}$ and let its unspliced semi-edge be $s_{0}$. By A3, every component has unspliced semi-edges, so every component but $C_{0}$ has at least two. Since A4 guarantees that each component has only finitely many semiedges, we arrange the lists so that, if $n<k$, then all the semi-edges in $C_{n}$ precede those in $C_{k}$ in the $s_{i}$-list.

Now splice as in the proof of Theorem 1 , using the $C_{n}$ in place of the $v_{n}$. Since no $C_{n}$ contains a cycle (the underlying graph of each $C_{n}$ being a star), we again obtain a forest; it remains to prove connectedness.

We claim that, after $n$ steps of the construction, the component containing $C_{0}$ consists of $C_{0}, C_{1}, \ldots, C_{n}$ and has at least one unspliced semi-edge, and that no $C_{m}$ with $m>n$ has yet been spliced to anything. This is obvious for $n=0$, that is, before the construction begins. If it is true for $n$, then at step $n+1$, the first unspliced semiedge will be in the component consisting of $C_{0}, \ldots, C_{n}$ (by our convention about the ordering of the semi-edges and the induction hypothesis), and it will be spliced to a semi-edge of the first $C_{k}$ not yet spliced to anything, namely, $C_{n+1}$. Thus, the component of $C_{0}$ after this step consists of $C_{0}, \ldots, C_{n+1}$. Furthermore, since $C_{n+1}$ 
had at least two unspliced semi-edges and since only one of them has been spliced, at least one remains. So the claim is true for $n+1$. The claim is therefore true, by induction on $n$, and connectedness of the final graph follows immediately.

(a) $\Rightarrow$ (c). Suppose there were a connected graph whose multiplicity function violates (c) while satisfying A1-A5. Since $m(1) \leqslant \aleph_{0}$ by A5, the sum $S=\sum_{d \geqslant 3}(d-2) \cdot m(d)$ must be finite for any such graph. Let $G$ be such a graph with the smallest possible value of $S$, and let $m$ be its multiplicity function. Of course, since (c) is violated, $m(1)$ is non-zero, so let $v_{0}$ be a vertex of degree 1 . Let $v_{1}$ be the unique vertex adjacent to $v_{0}$. If $v_{1}$ has degree 2 , let $v_{2}$ be the unique vertex other than $v_{0}$ adjacent to $v_{1}$. If $v_{2}$ has degree 2 , let $v_{3}$ be the unique vertex other than $v_{1}$ adjacent to $v_{2}$, etc. If any $v_{n}$ has degree $\neq 2$, then $v_{k}$ is undefined for all $k>n$. There are three cases to consider.

Case 1 , in which $v_{n}$ is defined for all $n$. Then, since the only vertices adjacent to $v_{n}$ are $v_{n \pm 1}$, and since $G$ is connected, the $v_{n}$ must be all the vertices of $G$. Thus, $m(1)=1$ and $m(d)=0$ for all $d \geqslant 3$. This contradicts the assumption that (c) is violated.

Case 2, in which there is a last $v_{n}$ and its degree is 1 . Then the set of $v_{n}$ again contains all vertices adjacent to any of its members, so, by connectedness, it exhausts $G$. This contradicts A2.

Case 3 , in which there is a last $v_{n}$, say $v_{k}$, and its degree is $\delta \geqslant 3$. Form a graph $G^{\prime}$ by deleting from $G$ the vertices $v_{0}, \ldots, v_{k-1}$ and the edges incident with them. The multiplicity function $m^{\prime}$ of $G^{\prime}$ differs from $m$ in that $m(1)$ has been reduced by 1 (because of $\left.v_{0}\right), m(2)$ has been reduced by $k-1$ (because of $\left.v_{1}, \ldots, v_{k-1}\right), m(\delta)$ has been reduced by 1 and $m(\delta-1)$ has been increased by 1 (because the degree of $v_{k}$ was changed from $\delta$ to $\delta-1$ ). The effect on both sides of the inequality (c) is to reduce them by 1 . So $G^{\prime}$ also violates (c), is clearly connected yet has a smaller $S$ than $G$. This contradicts the choice of $G$ as minimizing $S$.

Having obtained a contradiction in every case, we have established the implication from (a) to (c) and thus completed the proof of Theorem 2.

Remark. Compare condition (c) in Theorem 2 with the criterion

$$
2+\sum_{d \geqslant 3}(d-2) \cdot m(d)=m(1)
$$

for $m$ to be the multiplicity function of a finite tree; see $[2 ; p .62]$ for the characterization of the degree sequence of a finite tree. This condition suggests the following alternate proof that (a) implies (c) which was kindly supplied by the referee, using our notation as above.

(a) $\Rightarrow$ (c), another proof. Since $2+S \leqslant m(1)$, we can identify $2+S$ vertices of degree 1 in $G$. We will now construct a subgraph of $G$ which is a finite tree $T$ having just these $2+S$ vertices as its endpoints. To form $T$, we first use the connectedness of $G$ to join the chosen vertices pairwise with paths in $G$. From the union of the resulting paths we extract a spanning subtree and prune away any excess vertices of degree 1 to obtain $T$. 
Thus if $m_{T}$ is the multiplicity function of $T$, we have

$$
\begin{aligned}
2+S=m_{T}(1) & =2+\sum_{d \geqslant 3}(d-2) \cdot m_{T}(d) \quad\left(\text { by c }^{\prime}\right) \\
& \leqslant 2+\sum_{d \geqslant 3}(d-2) \cdot m(d)=2+S,
\end{aligned}
$$

the inequality arising because any vertex $v$ of $T$ not of degree 1 contributes $\operatorname{deg}_{T}(v)-2$ to the first sum and $\operatorname{deg}_{G}(v)-2$ to the second sum, while each vertex not in $T$ contributes zero to the first and at least zero to the second. It follows that the inequality cannot be strict, so every vertex of $T$ has the same degree in $T$ as in $G$. In other words, no vertex of $T$ has neighbors outside $T$. As $G$ is connected, $G=T$, contrary to the assumption that $G$ is infinite.

\section{Uncountable or non-locally finite graphs}

In this section, we assume the following, in addition to the assumptions A1-A3 that are in force throughout the paper.

A6. Either $m(d)>0$ for some infinite cardinal $d$, or $m(d)$ is uncountable for some $d$.

In other words, $m$ does not satisfy the hypothesis of Section 1 . We recall that the sum $\sum_{d} m(d)$ is denoted by $p$, and we write sup $d$ for the least upper bound of the cardinals $d$ for which $m(d)>0$. Thus, any graph with the multiplicity function $m$ has $p$ vertices and has degree sup $d$.

THEOREM 3. For a function $m$ satisfying $\mathrm{A} 1-\mathrm{A} 3$ and $\mathrm{A} 6$, the following are equivalent:

(a) $m$ is the multiplicity function of a graph;

(b) $m$ is the multiplicity function of a forest;

(c) $\sup d \leqslant p$.

THEOREM 4. For a function $m$ satisfying $\mathrm{A} 1-\mathrm{A} 3$ and $\mathrm{A} 6$, the following are equivalent:

(a) $m$ is the multiplicity function of a connected graph;

(b) $m$ is the multiplicity function of a tree;

(c) $\sup d=p$, and if $p$ is regular then $m(p)>0$.

In connection with condition (c) of Theorem 4, we recall that a cardinal number $q$ is said to be singular if it is the least upper bound (or, equivalently, the sum) of fewer than $q$ cardinals each of which is smaller than $q$; otherwise, $q$ is said to be regular. For example $\aleph_{0}$ is regular, and so are all successor cardinals; for these, the condition $m(p)>0$ follows from $\sup d=p$ (and A6 if $p=\aleph_{0}$ ). Other regular cardinals, called "weakly inaccessible cardinals", cannot be proved to exist on the 
basis of the usual axioms of set theory. It is only when $p$ is one of these that the second clause in Theorem 4 (c) makes any difference.

Proofs of Theorems 3 and 4. In both theorems, the implication from (b) to (a) is trivial. We prove (a) $\Rightarrow$ (c) first for Theorem 3 , then for Theorem 4 , and we prove (c) $\Rightarrow$ (b) first for Theorem 4, then for Theorem 3.

3(a) $\Rightarrow 3$ (c). Suppose $\sup d>p$. This means, by definition of $\sup d$, that $m(d)>0$ for some $d>p$. So, in any graph $G$ with multiplicity function $m$, there would have to be a vertex of degree $d$, which is more than the total number $p$ of vertices in $G$. This is obviously absurd.

$4(\mathrm{a}) \Rightarrow 4(\mathrm{c})$. Let $G$ be a connected graph with multiplicity function $m$. By what was just proved, $\sup d \leqslant p$. Suppose the inequality were strict. Let $v_{0}$ be any vertex of $G$, and inductively define $V_{n}$ to be the set of vertices adjacent to vertices in $\left\{v_{0}\right\} \cup V_{1} \cup \ldots \cup V_{n-1}$. By connectedness, $G$ is covered by $\left\{v_{0}\right\}$ and the $V_{n}$. Since each vertex has degree at most sup $d$, we have, by induction on $n$, that $\left|V_{n}\right| \leqslant \max \left(\sup d, \aleph_{0}\right)$, so that $p=|G| \leqslant \max \left(\sup d, \aleph_{0}\right)$. Since we are assuming $p>\sup d$, we must have $p \leqslant \aleph_{0}$ and sup $d$ finite. But this contradicts A6.

So $p=\sup d$. Suppose that $p$ is regular but the supremum is not attained, that is, $m(p)=0$. If $p$ were countable, A6 would be violated, so $p$ must be uncountable. Let $v_{0}, V_{1}, V_{2}, \ldots \ldots$ be as in the preceding paragraph; again, connectedness implies that they exhaust $G$. We show, by induction on $n$, that the set $W_{n}=\left\{v_{0}\right\} \cup V_{1} \cup \cup \ldots \cup V_{n-1}$ has cardinality strictly less than $p$. This is trivial for $n=1$ as $\left|W_{1}\right|=1$. Assume that it holds for $n$. The number of points in $V_{n}$ is bounded by the sum of the degrees of all the points in $W_{n}$ (since every vertex in $V_{n}$ is adjacent to one in $W_{n}$ ), which is the sum of fewer than $p$ cardinals each smaller than $p$. By regularity, $\left|V_{n}\right|<p$, so $W_{n+1}=W_{n} \cup V_{n}$ also has cardinality strictly less than $p$. This completes the proof that every $\left|W_{n}\right|<p$. Thus $G$ is the union of $\aleph_{0}$ sets of cardinality strictly less than $p$, which contradicts the fact that $p$ is regular and uncountable.

$4(\mathrm{c}) \Rightarrow 4(\mathrm{~b})$. Let $m$ satisfy $4(\mathrm{c})$. Proceeding as in Section 1, we start with a semigraph $P_{0}$ consisting of $p$ vertices, all of spliced degree 0 , of which $m(d)$ have unspliced degree $d$, and we try to splice all the semi-edges to produce a tree.

Suppose first that $m(p)>0$, so $P_{0}$ has a vertex $v_{0}$ of degree $p$. If $m(1)=p$, we splice (one semi-edge incident with) each vertex $v \neq v_{0}$ of degree $>1$ (if any) to (some semi-edge incident with) $v_{0}$, obtaining a semi-graph $P_{1}$ in which the component of $v_{0}$ has $p$ unspliced semi-edges (for whenever an unspliced semi-edge of $v_{0}$ is used to attach some $v$, this $v$ brings with it at least one other semi-edge that remains unspliced in $P_{1}$ ) and the other components are $p$ atoms of degree 1. Splice these atoms to the semi-edges of the component of $v_{0}$, and it is clear that a tree is obtained. Henceforth, we assume that $m(1)<p$. Splice the vertices of degree 1 to $v_{0}$; the resulting semi-graph $P_{1}$ is like $P_{0}$ except that, in place of $v_{0}$ and the vertices of degree 1, it has a component $C_{1}$ with $p$ unspliced semi-edges. Partition the other vertices (of which there are $p$, all of degree $\geqslant 2$ ) into $\aleph_{0}$ sets $A_{n}$ of $p$ vertices each. Obtain $P_{2}$ from $P_{1}$ by splicing one semi-edge from each vertex in $A_{1}$ to a semi-edge of $C_{1}$, using up all the latter semi-edges. Since each vertex in $A_{1}$ has degree at least 2 , it contributes an unspliced semi-edge to $P_{2}$. Thus, the nontrivial component $C_{2}$ of $P_{2}$, like $C_{1}$, has $p$ unspliced semi-edges. Next, form $P_{3}$ by splicing one semi-edge from each vertex in $A_{2}$ to a semi-edge of $C_{2}$, using up all of the latter. Continue in this 
manner until all the $A_{n}$ have been incorporated into $C_{n}$. The final result is connected (because each $C_{n}$ is) and is acyclic (because at no stage could a cycle be completed), so it is a tree, as required.

Now suppose $m(p)=0$. By assumption 4(c), $p$ must be singular. Let $p$ be the least upper bound (hence also the sum) of a set $\left\{q_{i} \mid i \in I\right\}$ of infinite cardinals $q_{i}<p$ indexed by a set $I$ of cardinality less than $p$. Since $p=\sup d$ by assumption, $P_{0}$ has a vertex $v_{0}$ of degree at least $|I|$ and, for each $i \in I$, a vertex $w_{i} \neq v_{0}$ of degree at least $q_{i}$. Splice one semi-edge from each $w_{i}$ to a semi-edge from $v_{0}$, forming a component $C_{0}$ with $p$ unspliced semi-edges. Now proceed as in the case $m(p)>0$, but use $C_{0}$ in place of the vertex $v_{0}$. The construction again produces a tree, as required.

$3(\mathrm{c}) \Rightarrow 3(\mathrm{~b})$. Let $m$ satisfy 3(c), and let $m^{\prime}$ be like $m$ except that $m^{\prime}(p)=m(p)+1$. Then $m^{\prime}$ satisfies 4(c), so it is the multiplicity function of a tree $G^{\prime}$. As $m^{\prime}(p) \geqslant 1, G^{\prime}$ has a vertex $v_{0}$ of degree $p$, and the other vertices have degrees as given by $m$. Remove $v_{0}$ and its $p$ incident semi-edges from $G^{\prime}$. The result is a semi-graph consisting of $p$ trees each with one unspliced semi-edge (where $v_{0}$ used to be attached). Since $p$ is infinite, the set of these semi-edges can be partitioned into $p$ pairs. Splice the two semi-edges in each pair to obtain a forest with multiplicity function $m$. This completes the proof of both theorems.

We note that in [2], Halin proves a more general result than (a) implies (c) in our Theorems 3 and 4.

Remark. Theorem 3 holds without the assumption A6, for if A6 fails, that is, if A4 and A5 hold, then 3(c) is trivially true while 3(a) and 3(b) are given by Theorem 1. Theorem 4, on the other hand, does not generalize in this way, since, when A6 fails, 4 (c) is false but 4(a) and 4(b) sometimes hold.

Our theorems imply that in contrast to the situation for finite graphs, the absence of cycles imposes no additional condition on the degree sequences (or multiplicity functions) of infinite graphs. It should be possible to use the methods of this paper to investigate other properties of infinite graphs, such as $n$-connectedness or unicyclicity, to determine what conditions they impose on the degree sequence. (That these two properties do impose additional conditions is clear; consider the degree sequence $(2,2,2, \ldots)$.) Other problems for further investigation include the characterization of degree sequences realized by a unique (up to isomorphism) graph and, more generally, an infinite analog to the well-developed theory of finite degree sequences.

\section{References}

1. S. L. Hakimi and E. F. Schmeichel, Graphs and their degree sequences: a survey, Lecture Notes in Mathematics 642 (Springer, Berlin, 1978), pp. 225-235.

2. R. Halin, "Simplicial decompositions of infinite graphs", Ann. Discrete Math., 3 (1978), 93-109.

3. F. Harary, Graph theory (Addison-Wesley, Reading, Ma., 1969).

4. F. Harary, "Demiarcs: an atomistic approach to relational systems and group dynamics", J. Math. Sociol., 1 (1971), 195-205.

5. F. Harary and R. Havelock, "Anatomy of a communication arc", Human Relations, 25 (1972), 413-426.

Department of Mathematics, The University of Michigan,

Ann Arbor,

Michigan 48109,

U.S.A. 